\title{
Tests of discrete symmetries with the neutral kaon system at the KLOE and KLOE-2 experiments
}

\author{
Aleksander Gajos ${ }^{1}$ (D) on behalf of the KLOE-2 Collaboration
}

Published online: 19 November 2018

(C) The Author(s) 2018

\begin{abstract}
The KLOE-2 experiment continues and extends the program of its predecessor KLOE in the field of discrete symmetry tests with the $\mathrm{K}$ meson system, among other studies which comprise light meson spectroscopy, dark matter searches and $\gamma \gamma$ physics. Together, KLOE and KLOE-2 have recorded the largest sample (almost $8 \mathrm{fb}^{-1}$ ) of $e^{+} e^{-}$collisions at the energy equal to $\phi$ meson mass, amounting to $2.4 \times 10^{10}$ of produced $\phi$ mesons. We report on the latest results and ongoing analyses of KLOE and KLOE- 2 concerning discrete symmetry tests and measurements of symmetry-violating observables. A measurement of the CPT violation sensitive asymmetry in semileptonic decays of $K_{S}$ with $1.7 \mathrm{fb}^{-1}$ of KLOE data will be presented, which improves the sensitivity w.r.t. previous measurements about twice. Moreover, status of direct tests of T and CPT in transitions of neutral kaons performed with the $\phi \rightarrow \mathrm{K}_{\mathrm{S}} \mathrm{K}_{\mathrm{L}} \rightarrow \pi e \nu, 3 \pi^{0}(2 \pi)$ processes will be reported, followed by the search of the $\mathrm{CP}$-violating decay $\mathrm{K}_{\mathrm{S}} \rightarrow 3 \pi^{0}$ using the data recently acquired by KLOE-2.
\end{abstract}

Keywords Discrete symmetries · CPT $\cdot$ CP $\cdot$ Neutral kaons

\section{Introduction}

Flavoured neutral mesons constitute powerful physical systems for testing fundamental discrete symmetries and their combinations such as CP and CPT. Among them, neutral kaons are extensively used to search for manifestations of symmetry violations as the symmetries are easily translated to constraints on the properties of this system [1].

Despite numerous experiments conducted with neutral $\mathrm{K}$ mesons to date, the potential of this system is far from being exploited. The KLOE experiment has contributed to determination of the neutral kaon properties as well as a number of tests of the CP and CPT symmetries [2]. However, the dataset collected by KLOE is still analysed with a view to obtaining more precise results, e.g. on the charge asymmetry in the semileptonic $\mathrm{K}_{\mathrm{S}}$ decays

This article is part of the Topical Collection on Proceedings of the 7th Symposium on Symmetries in Subatomic Physics (SSP 2018), Aachen, Germany, 10-15 June 2018

Guest Edited by Hans Ströher, Jörg Pretz, Livia Ludhova and Achim Stahl

Aleksander Gajos

aleksander.gajos@uj.edu.pl

1 Institute of Physics, Jagiellonian University, ul. Łojasiewicza 11, 30-348, Cracow, Poland 
and preforming new tests such as a first direct CPT test in transitions of neutral kaons. Moreover, the upgraded KLOE-2 detector has recently finished taking more than $5 \mathrm{fb}^{-1}$ of data which will allow for enhancing the precision of searches for the CP-violating decay $\mathrm{K}_{\mathrm{S}} \rightarrow 3 \pi^{0}$.

\section{The KLOE and KLOE-2 experiments}

KLOE and KLOE-2 operated at the DA $\Phi$ NE $\phi$-factory which collides electron and positron beams at $\sqrt{s} \approx 1020 \mathrm{MeV}=m_{\phi}$, producing predominantly $\phi$ mesons. Their decays into a pair of neutral kaons $\left(\mathrm{BR}\left(\phi \rightarrow \mathrm{K}^{0} \overline{\mathrm{K}}^{0}\right) \approx 34 \%\right)$ are recorded by the KLOE setup consisting of a large $(\mathrm{R}=2 \mathrm{~m})$ cylindrical drift chamber [3] surrounded by a sampling electromagnetic calorimeter with a coverage of $98 \%$ of $4 \pi$ [4]. KLOE-2 additionally features a cylindrical triple-GEM inner tracker [5] and calorimeters in the beamline region [6, 7] improving tracking and acceptance for particles at small polar angles. KLOE was taking data in the years 2001-2002 and 2004-2005, collecting $2.5 \mathrm{fb}^{-1}$ of $\phi$ decays. The KLOE-2 dataset was collected in 2014-2018 and amounts to over $5 \mathrm{fb}^{-1}$. Together, KLOE and KLOE-2 have provided the largest sample of $e^{+} e^{-} \rightarrow \phi$ events, corresponding to about $2.4 \times 10^{10}$ of produced $\phi$ mesons.

\section{Tests of CP and CPT with the charge asymmetry in $\mathrm{K}_{\mathrm{S}}$ semileptonic decays}

The charge asymmetry in the neutral kaon decays $\left(A_{S / L}\right.$ for $\mathrm{K}_{\mathrm{S}}$ and $\mathrm{K}_{\mathrm{L}}$ respectively) is related to parameters violating $\mathrm{CP}, \mathrm{CPT}$ and the $\Delta S=\Delta Q$ rule. While non-zero value of each of the asymmetries indicates CP violation, observation of a discrepancy between them would be a sign of CPT noninvariance.

The asymmetry in decays of $\mathrm{K}_{\mathrm{L}}$ has been measured by the $\mathrm{KTeV}$ experiment with a precision of $10^{-5}$ [8]. The best result in case of $K_{S}$ comes from the KLOE 2001-2002 measurement and its statistics-limited precision is 2 orders of magnitude below that of $\mathrm{K}_{\mathrm{L}}$ [9]. Recently, the full dataset of KLOE was therefore used to determine $A_{S}$ with a reduced statistical uncertainty.

Ks decay events were tagged by presence of a $\mathrm{K}_{\mathrm{L}}$ interaction in the calorimeter. Semileptonic decays were selected with an analysis of particle time of flight from the decay point to the calorimeter. A certain amount of background was allowed in the final event samples to perform a MC-based event counting. Semileptonic Ks decays were counted in a selected region of the following kinematic variable: $M^{2}=\left[E_{\mathrm{K}_{\mathrm{S}}}-E_{\pi}-E_{\nu}\right]^{2}-p_{e}^{2}$, which allowed for good separation of the signal and background regions as shown in Fig. 1.

MC-simulated $M^{2}$ distributions for signal $\left(\mathrm{K}_{\mathrm{S}} \rightarrow \pi^{ \pm} e^{\mp} \nu\right)$ and major background components were fitted to the spectrum obtained from experimental data. The numbers of semileptonic decay events obtained from the fit were $34579 \pm 251$ for $\mathrm{K}_{\mathrm{S}} \rightarrow \pi^{-} e^{+} v$ and $36874 \pm 255$ for $\mathrm{K}_{\mathrm{S}} \rightarrow \pi^{+} e^{-} \bar{v}$, resulting in the following value of the charge asymmetry:

$$
A_{S}=\left(-4.9 \pm 5.7_{\text {stat }} \pm 2.6_{\text {syst }}\right) \times 10^{-3} \text {. }
$$

The above result combined with the previous one also determined by KLOE constitutes the most precise measurement of $A_{S}$ available to date:

$$
A_{S}=\left(-3.7 \pm 5.0_{\text {stat }} \pm 2.6_{\text {syst }}\right) \times 10^{-3} \text {. }
$$



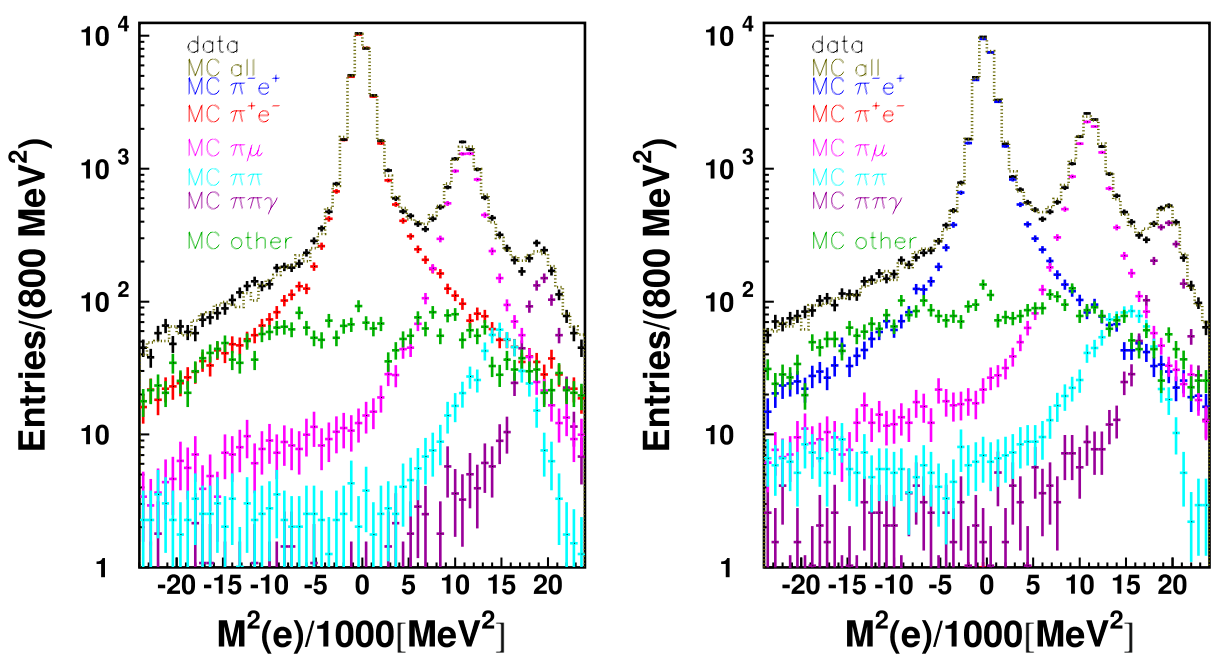

Fig. 1 Distributions of the kinematic variable used to separate signal and background for $\mathrm{K}_{\mathrm{S}} \rightarrow \pi^{+} e^{-} \bar{v}$ (left) and $\mathrm{K}_{\mathrm{S}} \rightarrow \pi^{-} e^{+} v$ (right) event counting. MC-simulated distributions for signal and major background components (color points) are normalized to experimental data (black histogram) to extract the number of signal events with each lepton charge [10]

\section{Direct test of T and CPT in transitions of neutral kaons}

A comparison of the rates of neutral mesons transitions between their flavour and $\mathrm{CP}$ eigenstates allows for a model independent test of the T and CPT symmetries [11, 12]. Such a test, to date performed only in the case of neutral B mesons where it delivered the first direct evidence of $\mathrm{T}$ violation [13], is pursued with the $\mathrm{K}^{0} \overline{\mathrm{K}}^{0}$ system at KLOE-2.

In this novel test, quantum entangled meson pairs are used to identify the initial state of a particle transition by the decay of its entangled partner, while the final state is tagged by semileptonic and hadronic decays into two and three pions. Two T-violating observables are determined as ratios of the rates of two classes of processes identified in the KLOE dataset: $\mathrm{K}_{\mathrm{S}} \mathrm{K}_{\mathrm{L}} \rightarrow \pi^{ \pm} e^{\mp} \nu, 3 \pi^{0}$ and $\mathrm{K}_{\mathrm{S}} \mathrm{K}_{\mathrm{L}} \rightarrow \pi^{+} \pi^{-}, \pi^{ \pm} e^{\mp} \nu$ :

$$
\begin{aligned}
& \mathrm{R}_{2}(\Delta t)=\frac{\mathrm{P}\left[\mathrm{K}^{0}(0) \rightarrow \mathrm{K}_{-}(\Delta t)\right]}{\mathrm{P}\left[\mathrm{K}_{-}(0) \rightarrow \mathrm{K}^{0}(\Delta t)\right]} \sim \frac{\mathrm{I}\left(\pi^{+} e^{-} \bar{\nu}, 3 \pi^{0} ; \Delta t\right)}{\mathrm{I}\left(\pi^{+} \pi^{-}, \pi^{-} e^{+} \nu ; \Delta t\right)}, \\
& \mathrm{R}_{4}(\Delta t)=\frac{\mathrm{P}\left[\overline{\mathrm{K}}^{0}(0) \rightarrow \mathrm{K}_{-}(\Delta t)\right]}{\mathrm{P}\left[\mathrm{K}_{-}(0) \rightarrow \overline{\mathrm{K}}^{0}(\Delta t)\right]} \sim \frac{\mathrm{I}\left(\pi^{-} e^{+} \nu, 3 \pi^{0} ; \Delta t\right)}{\mathrm{I}\left(\pi^{+} \pi^{-}, \pi^{+} e^{-} \bar{v} ; \Delta t\right)},
\end{aligned}
$$

where $\mathrm{I}\left(f_{1}, f_{2} ; \Delta t\right)$ denotes the number of recorded events characterized by a time-ordered pair of kaon decays $f_{1}$ and $f_{2}$ separate by an interval of proper kaon decay times $\Delta t$ [11]. A deviation of the asymptotic level of these ratios from unity for large transition times would be a T-violation manifestation.

Moreover, the concept of such test can be generalized to test the CPT symmetry through determination of the asymptotic level of the following double ratio:

$$
\frac{R_{2}^{C P T}}{R_{4}^{C P T}}=\frac{\mathrm{P}\left[\mathrm{K}^{0}(0) \rightarrow \mathrm{K}_{-}(\Delta t)\right] / \mathrm{P}\left[\mathrm{K}_{-}(0) \rightarrow \overline{\mathrm{K}}^{0}(\Delta t)\right]}{\mathrm{P}\left[\overline{\mathrm{K}^{0}} \rightarrow \mathrm{K}_{-}(\Delta t)\right] / \mathrm{P}\left[\mathrm{K}_{-}(0) \rightarrow \mathrm{K}^{0}(\Delta t)\right]} \stackrel{\Delta t \tau_{S}}{=} 1-8 \Re \delta_{K}-8 \Re x_{-},
$$




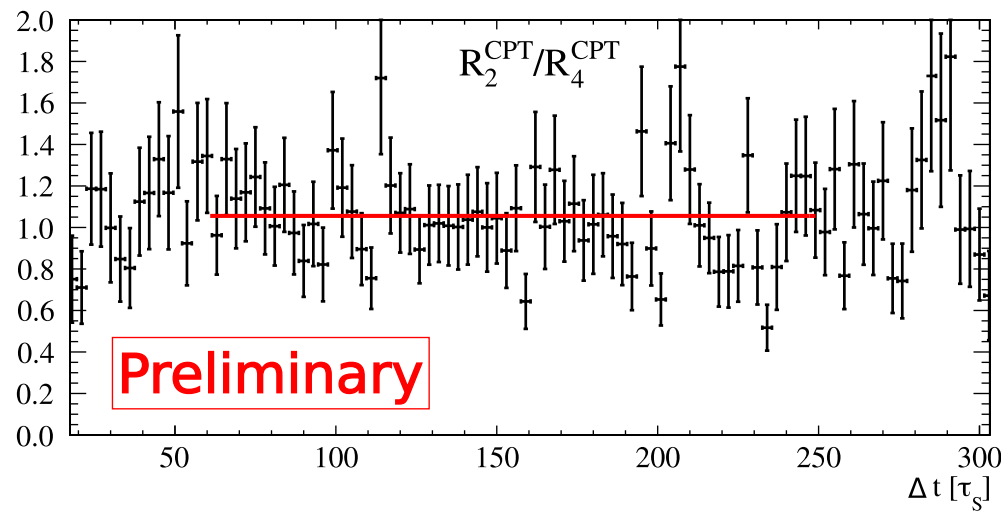

Fig. 2 Preliminary distribution of the CPT-violation sensitive double ratio of neutral kaon double decay rates as a function between entangled kaon decay times' difference $(\Delta t)$. The statistical uncertainty of the asymptotic level of this observable for $\Delta t \gg \tau_{S}$ (red line) amounts to 0.011 with the KLOE dataset

where the $\delta_{K}$ and $x_{-}$are parameters violating the CPT symmetry in $\mathrm{K}^{0} \overline{\mathrm{K}^{0}}$ mixing and the $\Delta S=\Delta Q$ rule, respectively. This double ratio constitutes a robust CPT-violation sensitive observable [12] which has never been measured to date.

The analysis tools required to determine both the above T-violation sensitive ratios and the CPT-violation sensitive double ratio are prepared and tested using the KLOE dataset. A preliminary distribution of the ratio defined in (5) obtained with $1.7 \mathrm{fb}^{-1}$ of KLOE data, is presented in Fig. 2. Although the uncertainty on the asymptotic level of these observables available with KLOE data is only at the percent level, the analysis is prepared with a view to its application to the larger sample of KLOE-2, which will allow for statistically significant tests of $\mathrm{T}$ and CPT at the $10^{-3}$ level of precision.

\section{Search for the CP-violating $K_{S} \rightarrow 3 \pi^{0}$ decay}

Another class of CP-violating phenomena in the neutral kaon system is constituted by the decays of $\mathrm{K}_{\mathrm{S}}$ into a CP-asymmetric state with three pions. While the $\mathrm{K}_{\mathrm{S}} \rightarrow \pi^{+} \pi^{-} \pi^{0}$ process contains both $\mathrm{CP}$-violating and conserving amplitudes, observation of the $\mathrm{K}_{\mathrm{S}} \rightarrow$ $3 \pi^{0}$ decay would be a clear signature of $\mathrm{CP}$ violation. The present best limit was set by $\mathrm{KLOE}$ at $\mathrm{BR}\left(\mathrm{K}_{\mathrm{S}} \rightarrow 3 \pi^{0}\right)<2.6 \times 10^{-8}$ [14], still almost an order of magnitude lower than the precision of the Standard Model prediction $\left(1.9 \times 10^{-9}\right)$. Therefore, the search for $\mathrm{K}_{\mathrm{S}} \rightarrow 3 \pi^{0}$ is already performed using the recently collected $5 \mathrm{fb}^{-1}$ of KLOE-2 data. The larger data sample and data analysis optimized with respect to the previous measurement are expected to increase the sensitivity for this rare decay beyond the level of $10^{-8}$.

\section{Summary and perspectives}

The measurements of the KLOE detector have recently delivered the most precise result on the charge asymmetry in the semileptonic decays of the short-lived neutral $\mathrm{K}$ meson, consistent with CPT conservation within the total uncertainty of $5.6 \times 10^{-3}$. The larger dataset collected recently by the upgraded KLOE-2 detector is expected to further improve 
the precision of this measurement to the level of $3 \times 10^{-3}$. The datasets of KLOE and KLOE2 are also used to perform first direct tests of the T and CPT symmetries in transitions of neutral kaons, expected to reach the precision of $10^{-3}$. Finally, the serch for the CP-violating decay $\mathrm{K}_{\mathrm{S}} \rightarrow 3 \pi^{0}$ is performed with the KLOE- 2 dataset. The expected sensitivity to this process should should allow KLOE-2 to approach the level of the present Standard Model predicion for $\mathrm{BR}\left(\mathrm{K}_{\mathrm{S}} \rightarrow 3 \pi^{0}\right)$.

Acknowledgements We warmly thank our former KLOE colleagues for the access to the data collected during the KLOE data taking campaign. We thank the DAФNE team for their efforts in maintaining low background running conditions and their collaboration during all data taking. We want to thank our technical staff: G.F. Fortugno and F. Sborzacchi for their dedication in ensuring efficient operation of the KLOE computing facilities; M. Anelli for his continuous attention to the gas system and detector safety; A. Balla, M. Gatta, G. Corradi and G. Papalino for electronics maintenance; C. Piscitelli for his help during major maintenance periods. This work was supported in part by the Polish National Science Centre through the Grants No. 2013/11/B/ST2/04245, 2014/14/E/ST2/00262, 2014/12/S/ST2/00459, 2016/21/N/ST2/01727, 2016/23/N/ST2/01293, 2017/26/M/ST2/00697.

Open Access This article is distributed under the terms of the Creative Commons Attribution 4.0 International License (http://creativecommons.org/licenses/by/4.0/), which permits unrestricted use, distribution, and reproduction in any medium, provided you give appropriate credit to the original author(s) and the source, provide a link to the Creative Commons license, and indicate if changes were made.

\section{References}

1. Di Domenico, A.: Handbook on neutral kaon interferometry at a $\Phi$-factory. Frascati Phys. Ser. 43, 1-38 (2007)

2. Bossi, F., De Lucia, E., Lee-Franzini, J., Miscetti, S., Palutan, M.: Precision Kaon and Hadron Physics with KLOE. Riv. Nuovo Cim. 31, 531-623 (2008). https://doi.org/10.1393/ncr/i2008-10037-9

3. Adinolfi, M., et al.: The tracking detector of the KLOE experiment. Nucl. Instrum. Methods A488, 5173 (2002). https://doi.org/10.1016/S0168-9002(02)00514-4

4. Adinolfi, M., Ambrosino, F., Antonelli, A., Antonelli, M., Anulli, F., et al.: The KLOE electromagnetic calorimeter. Nucl. Instrum. Methods A482, 364-386 (2002). https://doi.org/10.1016/S0168-9002(01) 01502-9

5. Balla, A., et al.: Status of the cylindical-GEM project for the KLOE-2 inner tracker. Nucl. Instrum. Methods A628, 194-198 (2011). https://doi.org/10.1016/j.nima.2010.06.315

6. Cordelli, M., et al.: CCALT: a crystal CALorimeter with timing for the KLOE-2 upgrade. Nucl. Instrum. Methods A718, 81-82 (2013). https://doi.org/10.1016/j.nima.2012.11.074

7. Cordelli, M., et al.: QCALT: a tile calorimeter for KLOE-2 experiment. Nucl. Instrum. Methods A617, 105-106 (2010). https://doi.org/10.1016/j.nima.2009.09.106

8. Alavi-Harati, A., et al.: A measurement of the $\mathrm{K}(\mathrm{L})$ charge asymmetry. Phys. Rev. Lett. 88, 181601 (2002). https://doi.org/10.1103/PhysRevLett.88.181601

9. Ambrosino, F., et al.: Study of the branching ratio and charge asymmetry for the decay $K(s) \rightarrow \pi e v$ with the KLOE detector. Phys. Lett. B636, 173-182 (2006). https://doi.org/10.1016/j.physletb.2006.03.047

10. Anastasi, A., et al.: Measurement of the charge asymmetry for the $K_{S} \rightarrow \pi e v$ decay and test of CPT symmetry with the KLOE detector. JHEP (in print). Preprint: arXiv:1806.08654 (2018)

11. Bernabeu, J., Di Domenico, A., Villanueva-Perez, P.: Direct test of time-reversal symmetry in the entangled neutral kaon system at a $\phi$-factory. Nucl. Phys. B868, 102-119 (2013). https://doi.org/10.1016/j.nuclphysb.2012.11.009

12. Bernabeu, J., Di Domenico, A., Villanueva-Perez, P.: Probing CPT in transitions with entangled neutral kaons. JHEP 10, 139 (2015). https://doi.org/10.1007/JHEP10(2015)139

13. Lees, J.P., et al.: Observation of time reversal violation in the $B^{0}$ Meson system. Phys. Rev. Lett. 109, 211801 (2012). https://doi.org/10.1103/PhysRevLett.109.211801

14. Babusci, D., et al.: A new limit on the CP violating decay $K_{S} \rightarrow 3 \pi^{0}$ with the KLOE experiment. Phys. Lett. B723, 54-60 (2013). https://doi.org/10.1016/j.physletb.2013.05.008 